\title{
Nonlinear Determination Method for Self-Heating Initiative Temperature of Sulfide Ores
}

\author{
Huimin Jin $\mathbb{D}^{1},{ }^{1}$ Wei Pan $\left(\mathbb{D},{ }^{1,2}\right.$ Xue Shen $\mathbb{D}^{1},{ }^{1}$ and Shuangyi Cheng $\mathbb{D}^{1}$ \\ ${ }^{1}$ School of Resources and Safety Engineering, Central South University, Changsha 410083, China \\ ${ }^{2}$ Key Laboratory of Ministry of Education of China for Efficient Mining and Safety of Metal Mines, \\ University of Science and Technology Beijing, Beijing 100083, China \\ Correspondence should be addressed to Wei Pan; panwei2012@csu.edu.cn
}

Received 28 April 2020; Revised 29 September 2020; Accepted 1 October 2020; Published 19 October 2020

Academic Editor: Chittaranjan Hens

Copyright (c) 2020 Huimin Jin et al. This is an open access article distributed under the Creative Commons Attribution License, which permits unrestricted use, distribution, and reproduction in any medium, provided the original work is properly cited.

\begin{abstract}
The purpose of this article is to explore a new method to determine the self-heating initiative temperature of sulfide ores for preventing spontaneous combustion of sulfide ores. Two typical ore samples with self-heating characteristics are studied by wavelet transform, recursive graph analysis, Hurst index extraction, and approximate entropy detection. On this basis, self-heating initiative temperature of sulfide ores was measured. The results indicate that the Hurst index of both ore samples in the experiment is greater than 0.5; moreover, the Hurst index in the later period of the experiment is greater than that of the earlier period. The self-heating of sulfide ores is an unsteady process with positive persistence and obvious mutation, so the possibility of self-heating of sulfide ores can be determined according to the change characteristics of Hurst index. The mutation times of the two samples are $864 \mathrm{~min}$ and $819 \mathrm{~min}$, respectively, reference values of selfheating initiative temperature are $219.4^{\circ} \mathrm{C}$ and $232.3^{\circ} \mathrm{C}$, and the average relative error is only $1.49 \%$, which are in good agreement with the measured values, and therefore, it provides basis for safety production of high-sulfur ore mine.
\end{abstract}

\section{Introduction}

Moist sulfide ores are usually easy to oxidize when there is oxygen in the air; the oxidation reaction is exothermic; if the reaction heat is not dissipated to the external environment completely, the temperature of the sulfide materials will increase; that is called self-heating [1-3]. Sulfide ores are always exposed to oxygen in the air rather than to any external heating during mining, mineral processing, storage, and transportation of related sulfide materials; this will eventually lead to serious potential hazards [3-8]. For example, when pyrite $\left(\mathrm{FeS}_{2}\right)$ is in contact with air and water, the following reactions will occur:

$$
\begin{aligned}
2 \mathrm{FeS}_{2}+7 \mathrm{O}_{2}+2 \mathrm{H}_{2} \mathrm{O}= & 2 \mathrm{FeSO}_{4}+2 \mathrm{H}_{2} \mathrm{SO}_{4}-2558.4 \mathrm{~kJ} \\
4 \mathrm{FeSO}_{4}+2 \mathrm{H}_{2} \mathrm{SO}_{4}+\mathrm{O}_{2}= & 2 \mathrm{Fe}_{2}\left(\mathrm{SO}_{4}\right)_{3}+2 \mathrm{H}_{2} \mathrm{O} \\
& -393.3 \mathrm{~kJ} \\
12 \mathrm{FeSO}_{4}+3 \mathrm{O}_{2}+6 \mathrm{H}_{2} \mathrm{O}= & 4 \mathrm{Fe}_{2}\left(\mathrm{SO}_{4}\right)_{3}+4 \mathrm{Fe}(\mathrm{OH})_{3} \\
& -762.5 \mathrm{~kJ}
\end{aligned}
$$

The self-heating initial temperature of sulfide ores is a comprehensive evaluation index of spontaneous combustion tendency of sulfide ores [9]. Once the self-heating of sulfide ores occurs, the spontaneous combustion fire will occur when the temperature of ore heap reaches the ignition point of ore. The prevention and control on spontaneous combustion of sulfide ores are always the main safety problem for sulfide ore deposits. It is very important to exactly measure the spontaneous combustion tendency of sulfide ores in order to protect the safety of miners' life, maintain the normal production of mine, and make good utilization of the natural resources [10-14].

According to the existing research literature about spontaneous combustion of sulfide ores, it can be found that spontaneous combustion tendency evaluation is a research hotspot. Thus, researchers have done a lot of studies on the self-heating characteristics of sulfide ores. Methods to identify the potential for self-heating of sulphides have been proposed [15-21], such as evaluation model based on entropy and set pair analysis theory [22], matter-element 
model [23], apparent activation energy method [24], uncertainty measurement model [25], and fisher discriminant analysis method [26]. In addition, Payant et al. [27] verified the hypothesis that galvanic interaction between some sulfide ores could accelerate self-heating effect; Somot and Finch [28] confirmed that a high pyrrhotite content could provide a more reducing environment favouring formation of hydrogen sulfide; Yang and $\mathrm{Wu}$ [29] found that exothermic reaction activity of sulfide ores is enhanced by all kinds of mechanical forces through the mechanical activation experiment.

Regrettably, as the self-heating experiment in laboratory is affected by multiple factors, such as ores particle size, ores moisture content, especially the heating rate of automatic heating incubator, the accuracy of self-heating initiative temperature measurement results needs to be validated. Therefore, self-heating initiative temperature becomes an important index for the evaluation of spontaneous combustion possibility of sulfide ores. Because of that need, it is worth studying to identify the mutation point in the process of self-heating and determine the reference value of the initiative temperature of self-heating by using the non-linear theory. Previous studies have shown that the self-heating of sulfide ores is a non-linear and unsteady evolution process. Li et al. [30] put forward a rheology and mutation model for spontaneous combustion of sulfide ore; Pan et al. [31] concluded that the self-heating process of sulfide ores can be divided into two stages, slow oxidation stage and rapid oxidation stage, and verified the conclusion through experiment.

In this study, we measured two typical sulfide ore samples; after extracting information from the measured temperature series based on wavelet analysis, the research series of ore samples are analyzed by using recurrence plot analysis, Hurst exponent extraction, and approximate entropy detection methods. Consequently, we proposed a new determination method for self-heating initiative temperature of sulfide ores based on non-linear theory and repeated the experiments to verify the accuracy of the conclusions in this paper.

\section{Self-Heating Experiment}

2.1. Experimental Materials. We used the multi-point sampling method to collect sulfide ore samples from a mine in China. According to the statistics in recent years, many spontaneous combustion cases have occurred: in 2004, when mining in the 910 layer, spontaneous combustion phenomenon is observed in the ores; in September 2007, fire areas expanded significantly. Consequently, the mine is forced to stop mining the orebody II.

In this experiment, two kinds of typical samples with self-heating tendency, named sample A and sample B, are selected as experimental materials; Figure 1 illustrates the typical micrograph of sulfide ores. Mineralogical analysis results indicate that the typical metal mineral of the ore samples is pyrite. The pyrite crystals are subhedral, euhedral, or cube-like, the maximum particle size of pyrite is about $3 \mathrm{~mm}$, and the average particle size is about $1 \mathrm{~mm}$. There are

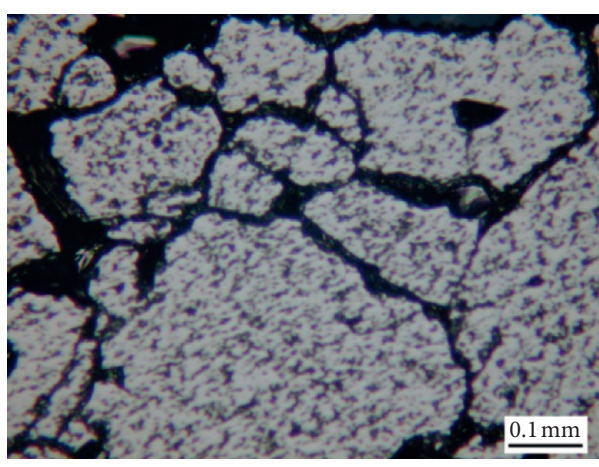

FIgURE 1: Typical micrograph of ore samples.

also a small amount of quartz and other gangue minerals found in the micrograph of the ore samples.

By means of chemical composition analysis, it is found that average sulfur content of the two samples is $41.77 \%$, greater than the average value of the mine $(29.41 \%)$. Sulfur content in sulfide ore is the decisive factor of spontaneous combustion of sulfide ore $[32,33]$. Therefore, both of sample A and sample B belong to the ore prone to spontaneous combustion.

2.2. Experimental Scheme. At room temperature, sulfide ores could slightly oxidize; in order to speed up the oxidation process, ore samples are put into the reactor in the automatic heating incubator. Self-heating initiative temperature of sulfide ores is defined as the minimum ambient temperature that can lead to rapid oxidation of sulfide ore [34]. If there is an obvious self-heating tendency in the ore samples, the temperature of the ore sample can increase rapidly and eventually exceed the ambient temperature of the automatic heating incubator. The crossing point temperature is determined as self-heating initiative temperature of ore sample, the main experimental parameters are listed in Table 1, and the experimental device is shown in Figure 2.

2.3. Experimental Results. It can be found from Figure 3 that the self-heating initiative temperatures of the two samples are $219.7^{\circ} \mathrm{C}$ and $239.1^{\circ} \mathrm{C}$, respectively. According to the definition of self-heating initiative temperature and the measured self-heating curves of the two samples, we can find that both samples have obvious self-heating phenomenon and the corresponding self-heating initiation time is $855 \mathrm{~min}$ and $867 \mathrm{~min}$, respectively.

\section{Research Methods}

Four main research methods, including wavelet transform [35], recurrence plot analysis [36], Hurst exponent extraction [37], and approximate entropy detection [38], are adopted in this work. Wavelet transform is used to remove the influence of automatic heating incubator on self-heating process of sulfide ores. Through recurrence plot analysis, mutation characteristics of self-heating of ore samples are identified qualitatively. By comparing the Hurst exponent of the earlier period and the later period during self-heating 
TABle 1: Main experimental parameters.

\begin{tabular}{lccccc}
\hline Ores diameter $(\mathrm{mm})$ & Mass $(\mathrm{g})$ & Moisture content $(\%)$ & Initial temperature $\left({ }^{\circ} \mathrm{C}\right)$ & $\begin{array}{c}\text { Average heating rate } \\
\left({ }^{\circ} \mathrm{C} \cdot \mathrm{min}^{-1}\right)\end{array}$ & $\begin{array}{c}\text { Temperature record interval } \\
(\mathrm{min})\end{array}$ \\
\hline$<0.2$ & 100.0 & 5.0 & $36.5 \sim 49.5$ & 1.0 & 3.0 \\
\hline
\end{tabular}

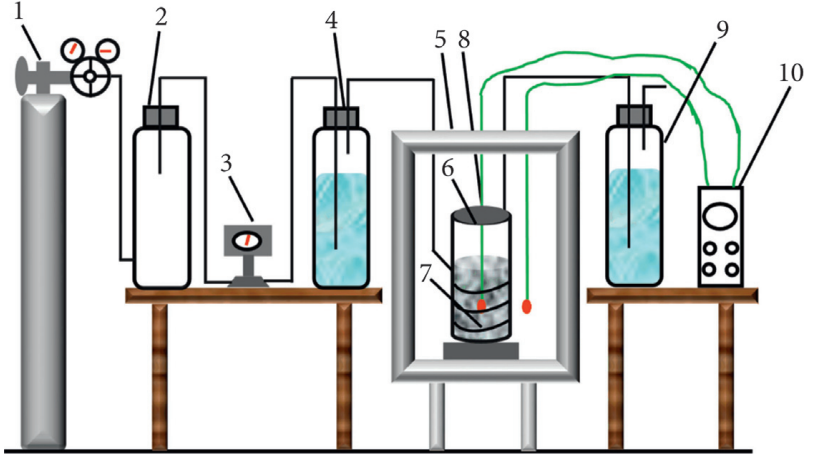

Figure 2: Experimental apparatus: 1: oxygen cylinder; 2: buffering bottle; 3 : flow meter; 4 : humidifier; 5 : automatic heating incubator; 6: reactor; 7: ores; 8: temperature probe; 9: poisonous gas absorption bottle; 10: automatic temperature recorder.

experiment, mutation characteristics of self-heating are quantitatively identified. With the approximate entropy detection method, the mutation points and reference values of self-heating initiative temperature are determined. The concrete research process is shown in Figure 4.

\section{Calculation Results Analysis}

4.1. Wavelet Transform of the Measured Temperature Series. Wavelet analysis is derived from the expansion and translation of functions; it is the direct result of the development of Fourier analysis, Gabor analysis, and short-term Fourier analysis. For any signal function $f(x)$ with finite energy, its continuous wavelet transform formula is [39]

$$
W_{f}(a, b)=\frac{1}{\sqrt{a}} \int_{R} f(x) \varphi \frac{x-b}{a} \mathrm{~d} x .
$$

In this formula, $\mathbf{W}_{\mathbf{f}}(\mathbf{a}, \mathbf{b})$ is the wavelet transform coefficient, $\mathbf{f}(\mathbf{x})$ is the signal to be transformed, $\varphi(\mathbf{x})$ is the base wavelet, $\mathbf{a}$ is the scale factor of the wavelet, and $\mathbf{b}$ is the translation factor of the wavelet.

The key of wavelet analysis is to select the appropriate wavelet function. Firstly, different wavelet functions are used to decompose the corresponding sequence of each measuring point into two parts: low frequency and high frequency. Then, the eigenvalues $\left(C_{v}, r_{1}\right)$ of the low-frequency reconstruction sequence and the original sequence are compared, and if the selected wavelet function is appropriate, the eigenvalues between the low-frequency reconstruction sequence and the original sequence should conform to the following criteria:

(1) Because the complex information in the self-heating process of ore heap is removed, the $C_{v}$ value of lowfrequency reconstruction sequence should be reduced compared with the original sequence.
(2) The interference of complex information in the selfheating process of ore heap will weaken the correlation of the original sequence. After eliminating these interferences, the $r_{1}$ value of low-frequency reconstruction sequence should be increased.

According to the main characteristic values of the corresponding series in Figure 5, it can be seen from the results of wavelet function optimization that the wavelet function bior3.1 is suitable for samples temperature measurement series. Obviously, both for the two samples, the autocorrelation coefficient $r_{1}$ of low-frequency reconstructed series is greater than that of the original series, but the variation coefficient $C_{v}$ of low-frequency reconstructed series is less than that of the original series (because interference of the complicated self-heating information will weaken the correlation of the original series, resulting in the increase of degree of change).

Through wavelet decomposition and reconstruction with bior3.1, research series of the two samples are obtained. Wavelet transform for the measured temperature increment series of the two samples is displayed in Figure 6. It is found that, compared with the original series, research series have more complex details and show sharp fluctuations.

4.2. Recurrence Plot Analysis. The reconstruction of phase space is the basis for the analysis of recurrence plot for nonlinear time series. According to the embedding theorem proposed by Takens [40], the phase space of non-linear time series $\mathbf{X}_{i}$ is reconstructed by selecting appropriate time delay $\tau$ and embedding dimension $\mathbf{m}$. At this time, the reconstructed phase space is equivalent to the original dynamic system and has the same topological structure.

Taking any sequence $\left\{\mathbf{x}_{\mathbf{i}}\right\}_{\mathbf{i}=\mathbf{1}}^{\mathbf{n}}$ as an example, the reconstructed phase space elements are as follows:

$$
\mathbf{X}_{\mathbf{i}}=\left\{\mathbf{x}_{\mathbf{i}}, \mathbf{x}_{\mathbf{i}+\tau}, \ldots, \mathbf{x}_{\mathbf{i}+(\mathbf{m}-1) \tau}, \quad \mathbf{i}=1,2, \ldots, \mathbf{N}\right\} .
$$

In this formula, $\mathbf{m}$ is the embedding dimension; $\boldsymbol{\tau}$ is the delay time; $\mathbf{N}=\mathbf{n}-(\mathbf{m}-\mathbf{1})$ is the reconstructed phase number; and $\mathbf{n}$ is the sequence length.

From the equation, the trajectory of the dynamic system in the reconstructed phase space is obtained as follows:

$$
\begin{aligned}
\mathrm{X}_{1} & =\left(x_{1}, x_{1+\tau}, \ldots, x_{1+(m-1) \tau}\right), \\
\mathrm{X}_{2} & =\left(x_{2}, x_{2+\tau}, \ldots, x_{2+(m-1) \tau}\right), \\
\mathrm{X}_{3} & =\left(x_{3}, x_{3+\tau}, \ldots, x_{3+(m-1) \tau}\right), \\
& \vdots \\
\mathrm{X}_{\mathrm{N}} & =\left(x_{N}, x_{N+\tau}, \ldots, x_{N+(m-1) \tau}\right) .
\end{aligned}
$$




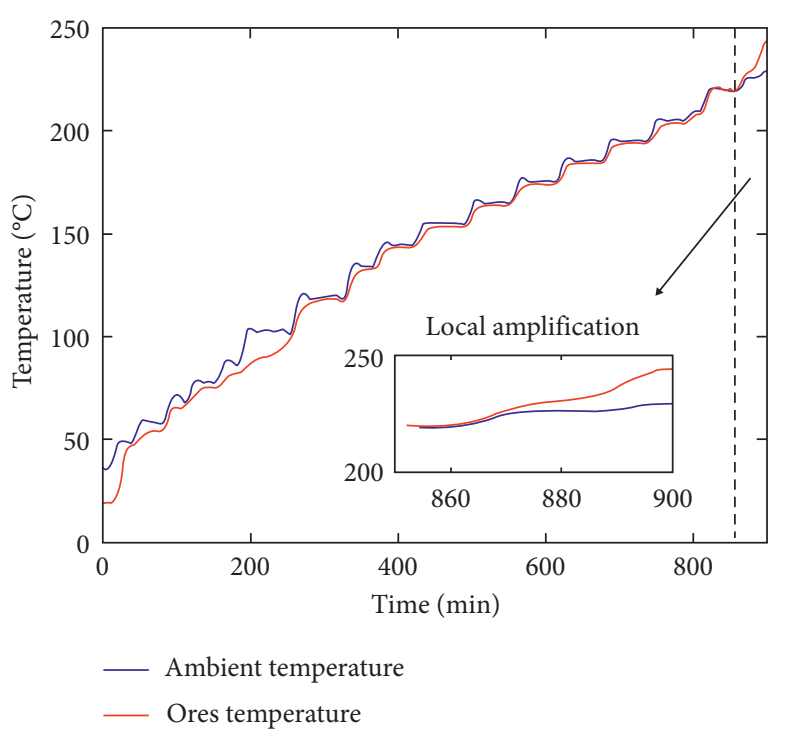

(a)

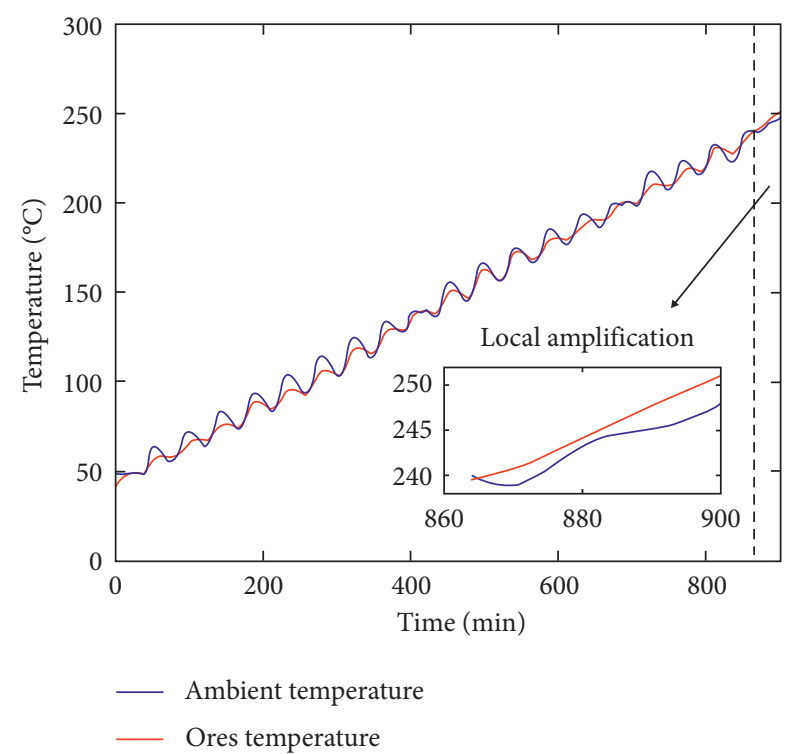

(b)

Figure 3: Measured self-heating curves of ore samples: (a) sample A; (b) sample B.

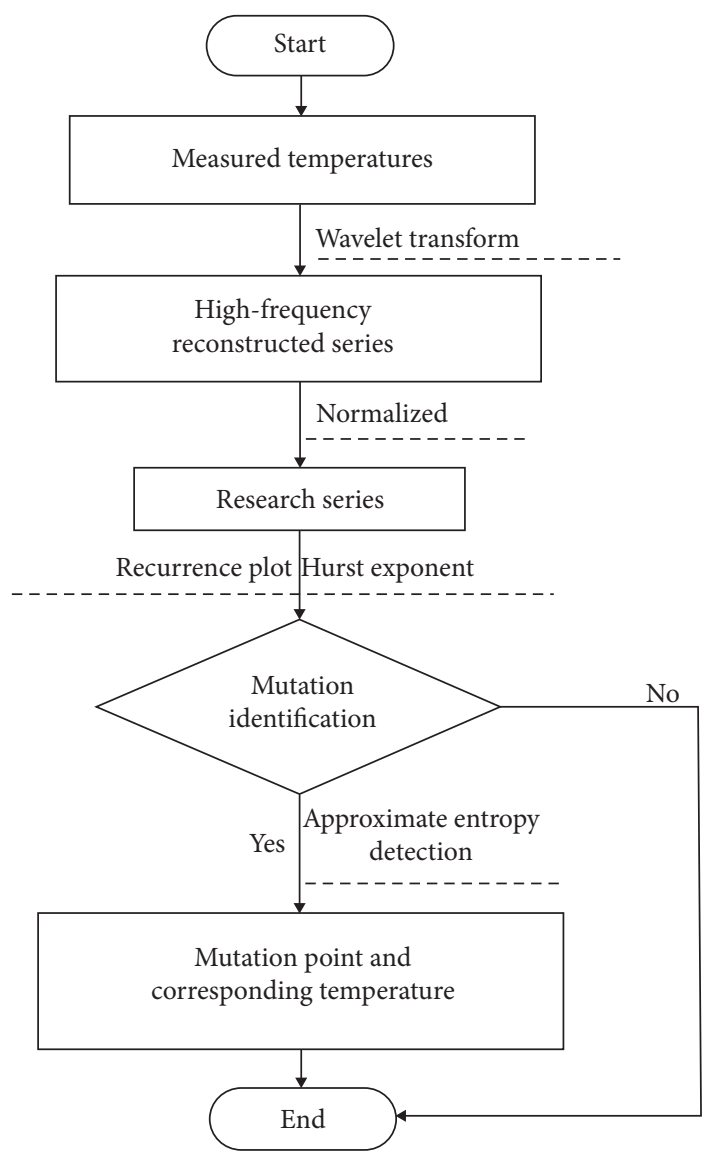

FIgURE 4: Schematic diagram of research process.

When reconstructing the phase space, the complex autocorrelation method is used to determine the delay time. The m-dimensional depolarization complex autocorrelation method is defined as follows [41]:

$$
\begin{aligned}
\mathbf{C}_{\mathrm{xx}}^{\mathbf{m}}(\boldsymbol{\tau}) & =\frac{1}{\mathbf{n}} \sum_{\mathbf{i}=\mathbf{1}}^{\mathbf{n}} \sum_{\mathbf{j}=\mathbf{1}}^{\mathbf{m}-\mathbf{1}}\left\{\left[\mathbf{x}_{\mathbf{i}}-\bar{x}\right]\left[\mathbf{x}_{\mathbf{i}+\mathbf{j} \tau}-\bar{x}\right]\right\} \\
& =\mathbf{R}_{\mathrm{xx}}^{\boldsymbol{\tau}}(\boldsymbol{\tau})-(\mathbf{m}-\mathbf{1})(\bar{x})^{2} .
\end{aligned}
$$

In this formula, $\overline{\mathbf{x}}$ is the mean value of the sequence; $\mathbf{R}_{\mathbf{x x}}^{\tau}(\tau)=\mathbf{1} / \mathbf{n} \sum_{\mathbf{i}=\mathbf{1}}^{\mathbf{n}} \sum_{\mathbf{j}=\mathbf{1}}^{\mathbf{m}-\mathbf{1}} \mathbf{x}_{\mathbf{i}} \mathbf{x}_{\mathbf{i}+\mathbf{j} \tau}$. The first zero of $\mathbf{C}_{\mathbf{x x}}^{\mathbf{m}}(\tau)$ is selected as the delay time $\tau$.

The analysis steps of recurrence plot analysis are as follows [42]:

(1) Let the reconstructed phase space elements be

$$
\mathbf{X}_{\mathbf{i}}=\left\{\mathbf{x}_{\mathbf{i}}, \mathbf{x}_{\mathbf{i}+\tau}, \ldots, \mathbf{x}_{\mathbf{i}+(\mathbf{m}-1) \tau}, \quad \mathbf{i}=1,2, \ldots, \mathbf{N}\right\}
$$

where $\mathbf{m}$ is the embedding dimension; $\boldsymbol{\tau}$ is the delay time; $\mathbf{N}=\mathbf{n}-(\mathbf{m}-\mathbf{1}) \tau$ is the reconstructed phase number; and $\mathbf{n}$ is the sequence length.

(2) Calculate the distance between $\mathbf{X}_{\mathbf{i}}$ and $\mathbf{X}_{\mathbf{j}}: \delta_{\mathbf{i j}}=\mathbf{X}_{\mathbf{i}}-\mathbf{X}_{\mathbf{j}}$.

(3) Given a distance $\mathbf{r}$, when $\delta_{\mathbf{i j}}<\mathbf{r}$, make a point at $(\mathbf{i}, \mathbf{j})$, and the resulting graph is called recurrence plot.

According to the definition of recurrence plot, it describes how the reconstructed trajectory recurses or repeats itself, and reveals the time related information of the system. Based on the complex autocorrelation method, the delay time $\tau$ of the standardized sequence is calculated. According to the theory of phase space reconstruction, the dimensional phase space $\mathbf{m}$ of the sequence is reconstructed. The basic idea of estimating the optimal delay time $\tau$ is to make the reconstructed phase space vector component $\mathbf{x}_{\mathbf{i}}, \mathbf{x}_{\mathbf{i}+\tau}$ independent of each other and not completely unrelated, so that they can become independent coordinates in phase space. 

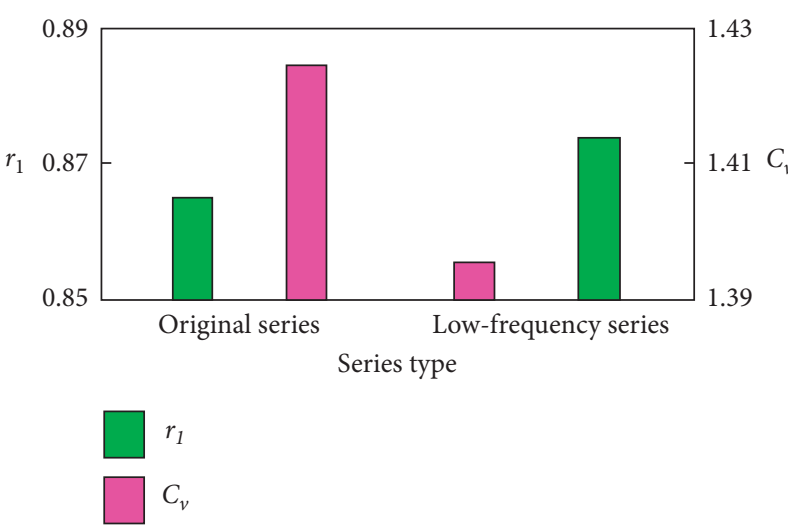

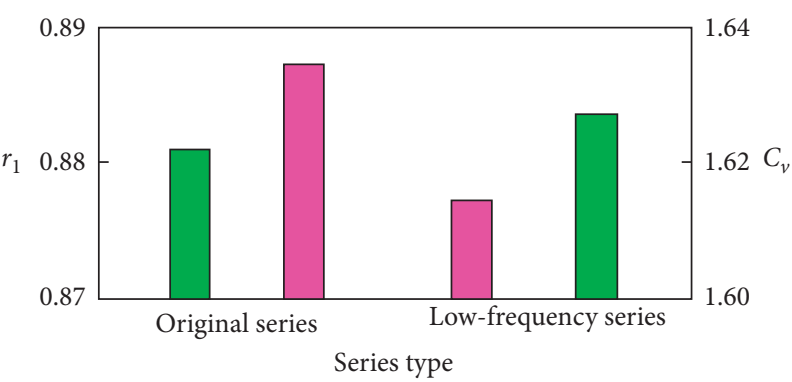

$r_{1}$

$C_{v}$

(a)

(b)

FIGURE 5: Main characteristic values of corresponding series: (a) sample A; (b) sample B.

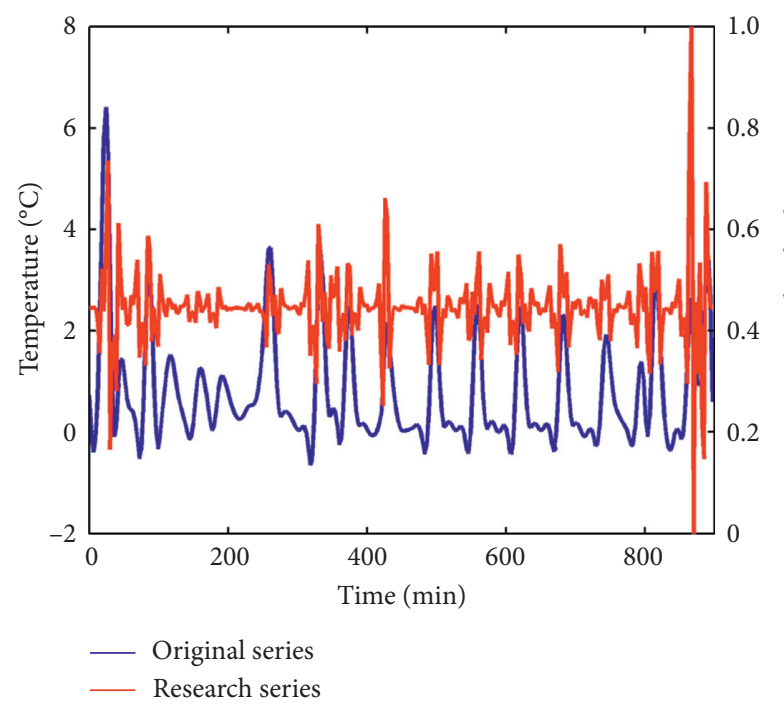

(a)

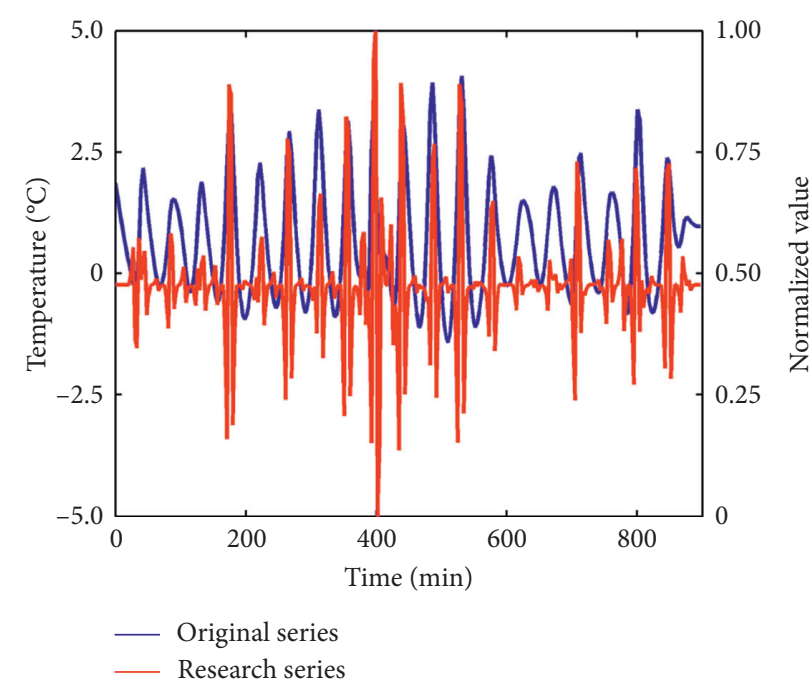

(b)

Figure 6: Wavelet transform for temperature increment series of ore samples: (a) sample A; (b) sample B.

Appropriate delay time is critical to recurrence plot analysis. In this research, delay time of the two samples is determined based on the modified autocorrelation function method [43]. The calculation results of delay time are shown in Figure 7. It can be found that autocorrelation function values of the two samples change from positive to negative when delay time increases from $3 \mathrm{~min}$ to $6 \mathrm{~min}$, which indicates that their correlation trends have changed during this period. According to the determination criterion, the optimal delay time of the two samples is equal to $3 \mathrm{~min}$.

The recurrence plots of the two samples are demonstrated in Figure 8. It is clear that self-heating process of sulfide ores contains mutation structures because there are large areas of white blocks or bands in recurrence plots. Compared with sample B, mutation structure of sample A is more complicated. The possible reason is that self-heating initiative temperature of the sample A is relatively low. Under the same experimental conditions, complexity of the self-heating process of sample A is more than that of sample B.
4.3. Hurst Exponent Analysis. Hurst exponent is an indicator of temporal sequential correlation and trend intensity. By comparing Hurst exponents of different periods, the unsteady self-heating process of sulfide ores can be quantitatively identified.

The Hurst exponent calculation results of the two samples in some periods are shown in Figure 9, where R/S and $\mathrm{n}$ are separately average rescaled range and subinterval length. The slope of the fitting line in the figure is the calculation value of Hurst exponent. It is found that all the Hurst exponents are more than 0.5. According to the definition of Hurst exponent [44] $(H=0.5$ refers to the time series which can be described by random walk "Brownian motion"; $0<H<0.5$ refers to the weakening of memory/ anti-persistence, that is, the process of mean reversion; $0.5<H<1$ refers to memory enhancement/persistence, that is, the time series of long-term memory), it can be concluded that self-heating process of sulfide ores has a positive continuity, which accords with the positive acceleration 


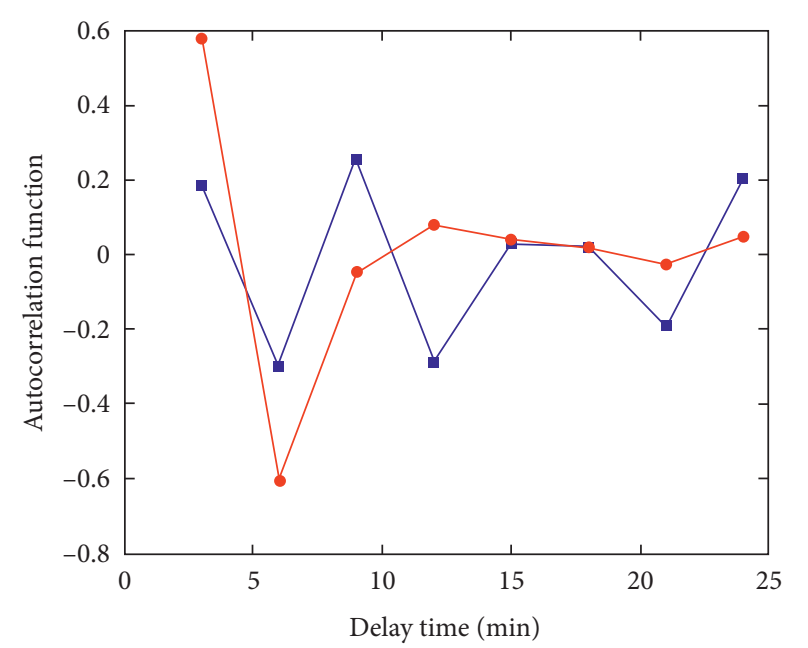

$\rightarrow$ Sample A

$\rightarrow$ Sample B

Figure 7: Calculation results of delay time of ore samples.

characteristics of self-heating reaction. It is also found that Hurst exponent of the later period is generally greater than that of the earlier period, especially in 750 897 min; the Hurst exponent increases of the two samples are $20.86 \%$ and $5.31 \%$, respectively. This is because ores temperature increases with time in self-heating experiment, which accelerates the self-heating and leads to the increase of positive correlation. Thus, the self-heating possibility of sulfide ores can be determined based on the variation characteristics of Hurst exponent. If Hurst exponent increases with time during the self-heating experiment, it means that the oxidation rate of sulfide ores can be accelerated and finally ores can produce obvious self-heating under the heating condition; otherwise, it means that ores cannot show obvious self-heating tendency.

4.4. Approximate Entropy Detection. To obtain the mutation point and reference value of self-heating initiative temperature of each sample with approximate entropy detection method, the sliding window width and sliding step length are set as $297 \mathrm{~min}$ and $3 \mathrm{~min}$, respectively.

Figure 10 illustrates mutation detection results of the two samples. It is clear that there are obvious mutation points on the approximate entropy curves. It is also found that approximate entropy changes irregularly at slow oxidation stage. The reason is that ambient temperature of the automatic heating incubator at this stage is not enough to induce sulfide ores to produce rapid self-heating. Ores self-heating effect is not significant so that the complexity change shows the sign of fluctuation. At rapid oxidation stage, the reason why the approximate entropy increases first and then decreases is that, in the early stage of rapid oxidation, the oxidation rate of sulfide ore increases rapidly due to the energy accumulation and activation. Ores temperature reaches the critical value of rapid self-heating. Therefore, the system complexity increases gradually at the later stage of rapid oxidation; as the surface of ore particles has been oxidized, the system complexity reduces gradually. So, it can be inferred that the complexity trend of oxidation process of sulfide ores includes three stages: relatively stable stage, gradually increasing stage, and gradually decreasing stage. At the gradually increasing stage, sulfide ores generate obvious self-heating.

For each sample, ambient temperature of the automatic heating incubator at mutation time is taken as the reference value of self-heating initiative temperature. Comparisons of the reference and measured values of self-heating initiative temperature are listed in Table 2. It is found that the reference values are consistent with measured values, and the average relative error is only $1.49 \%$. Therefore, it is feasible to use the method based on non-linear theory to determine the self-heating initiative temperature.

\section{Discussion}

Finding the crossing point accurately is the key to determine self-heating initiative temperature of sulfide ores. But actually ores temperature usually cannot exceed the ambient temperature in the experiment because of the low heat liberation and other influencing factors. In this case, selfheating experiments will be carried out several times under fine-tuned experimental conditions. For those sulfide ores without self-heating tendency, repeated experiments will result in a great deal of waste of manpower and material. Moreover, the difference of heating rate has certain effect on the measured results. For instance, when the heating rate is overtop, the ore temperature is usually lower than the ambient temperature. Consequently, the measured selfheating curves often have no crossing point even if the selfheating initiative temperature could be measured; it is usually greater than the actual value. When the heating rate is too low, the samples might be completely oxidized at slow oxidation stage. Under the circumstances, obvious selfheating phenomenon usually cannot appear in the sulfide ores. For example, when the average heating rate is $0.3^{\circ} \mathrm{C} \cdot \mathrm{min}^{-1}, 0.5^{\circ} \mathrm{C} \cdot \mathrm{min}^{-1}, 0.8^{\circ} \mathrm{C} \cdot \mathrm{min}^{-1}$, and $1.3^{\circ} \mathrm{C} \cdot \mathrm{min}^{-1}$ in this experiment, there are no crossing points on the measured self-heating curves of the two samples. Thus, selfheating initiative temperature of the two samples could not be measured in this case.

From the view of complexity variation in self-eating process, the non-linear method to determine the reference value of self-heating initiative temperature of sulfide ores is put forward in this work. This method includes three steps: first, preprocessing of the measured temperature series based on wavelet technology, which could eliminate the influence of automatic heating incubator on self-heating process of sulfide ores to a certain extent; second, mutation identification of self-heating process with recurrence plot method and Hurst exponent method, which could identify the ore samples without self-heating tendency and reduce workload of repeated experiments; third, mutation detection of selfheating process using approximate entropy method, which could obtain the mutation time and reference value of selfheating initiative temperature. The practical application 


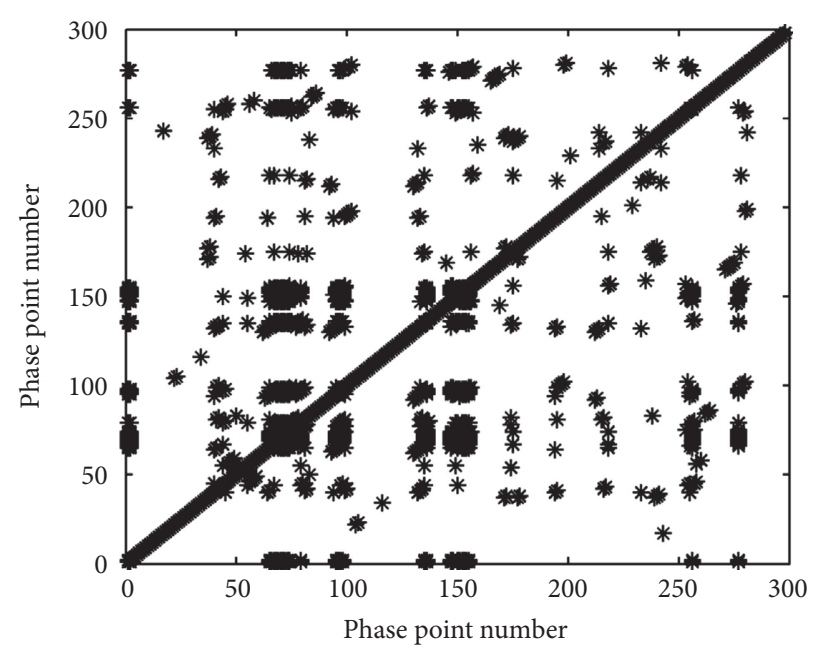

(a)

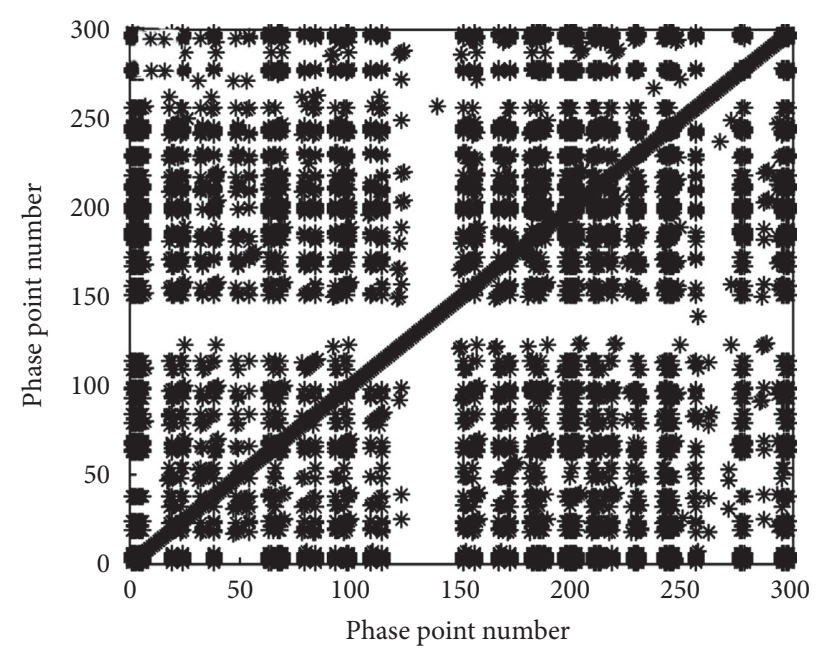

(b)

FIgURE 8: Recurrence plots of ore samples: (a) sample A; (b) sample B.

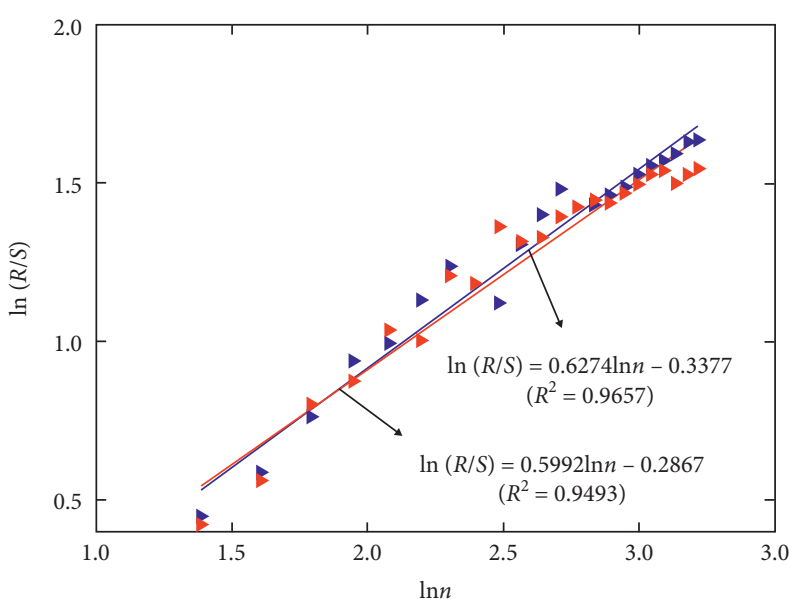

- Calculated values of sample A — Fitting line of sample A

- Calculated values of sample B _ F Fitting line of sample B

(a)

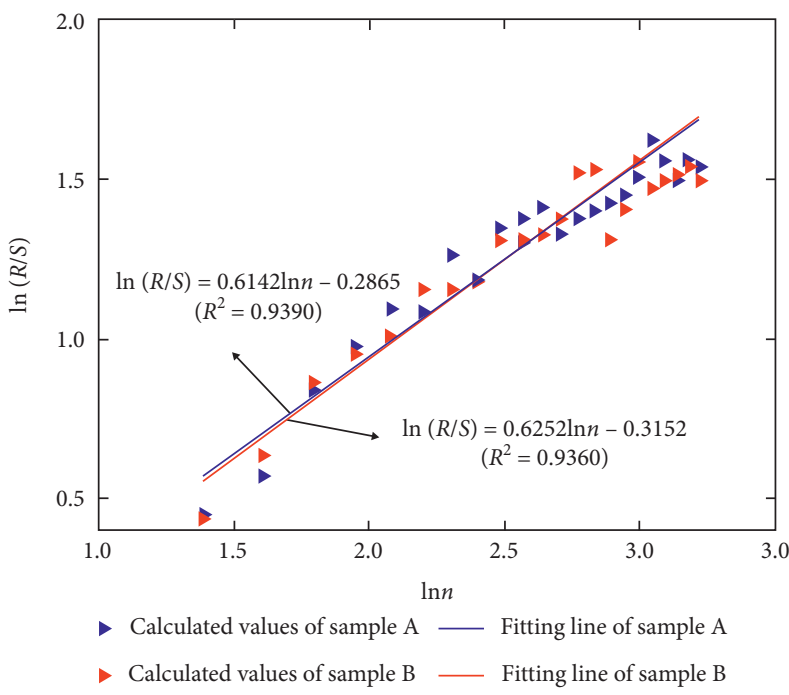

(c)

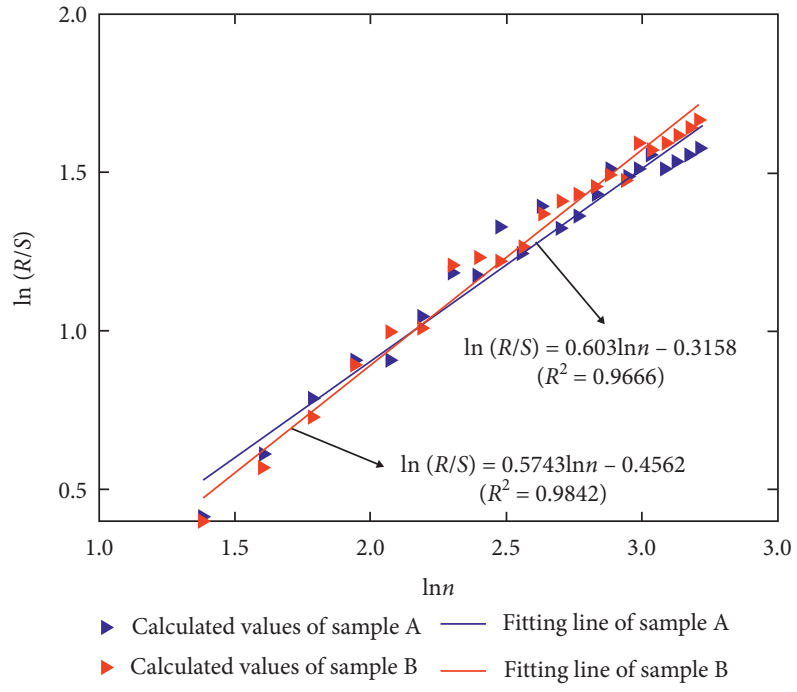

(b)

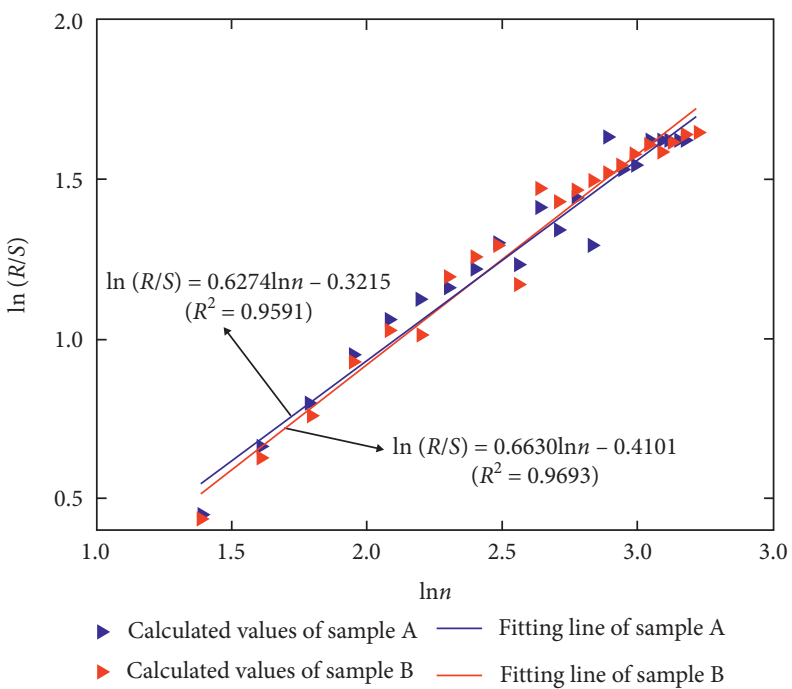

(d)

FIGURE 9: Continued. 


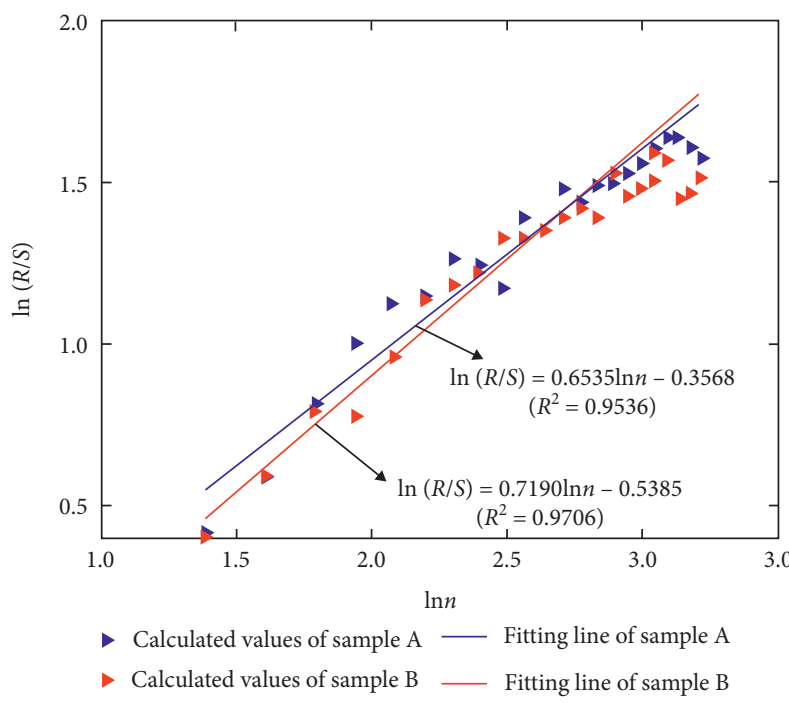

(e)

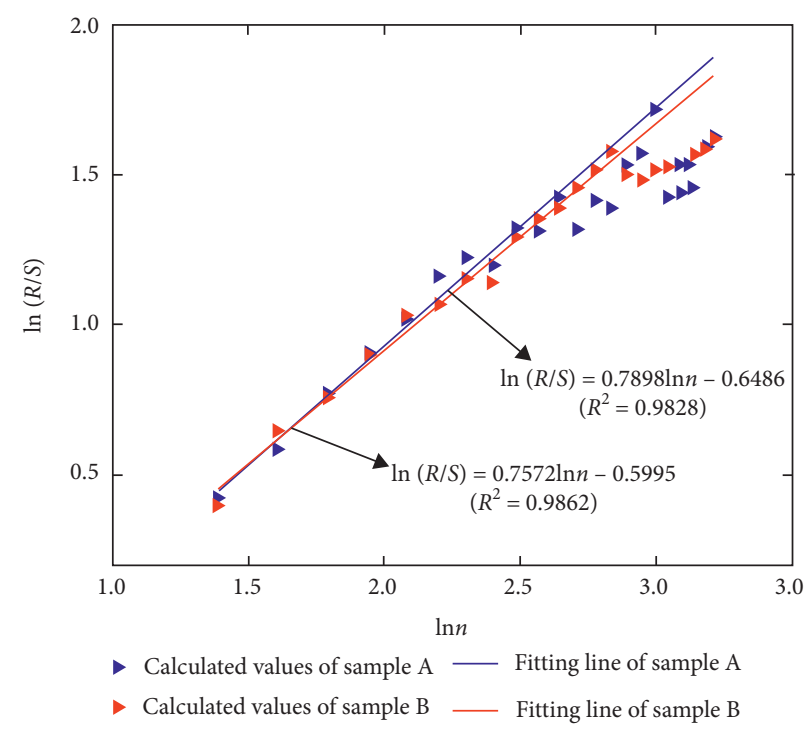

(f)

Figure 9: Hurst exponent calculation results of ore samples in some periods: (a) 0 147 min; (b) 150 297 min; (c) 300 447 min; (d) 450 597 min; (e) 600 747 min; (f) 750 897 min.

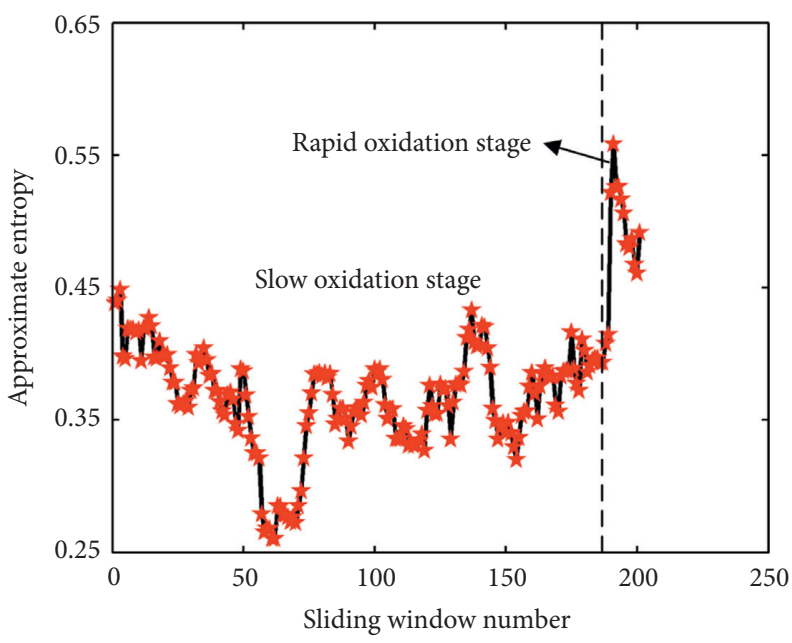

(a)

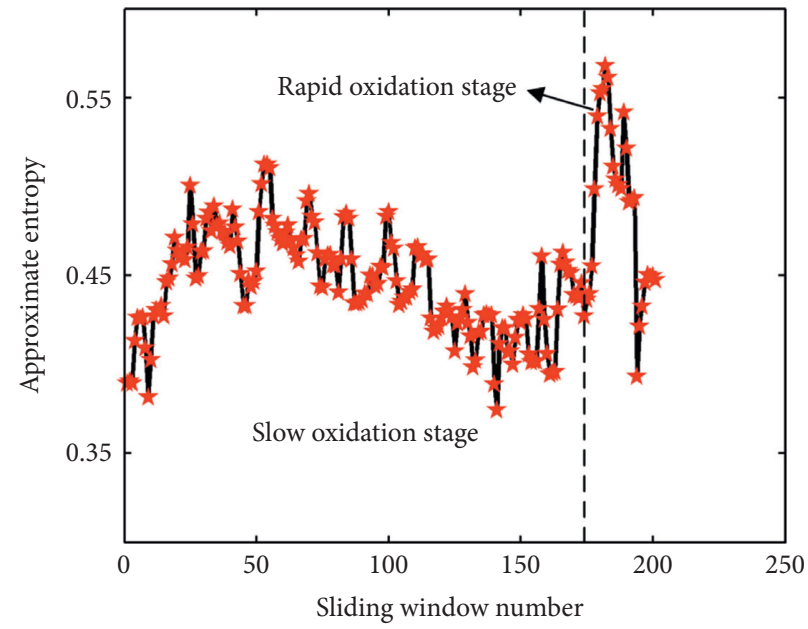

(b)

Figure 10: Continued. 


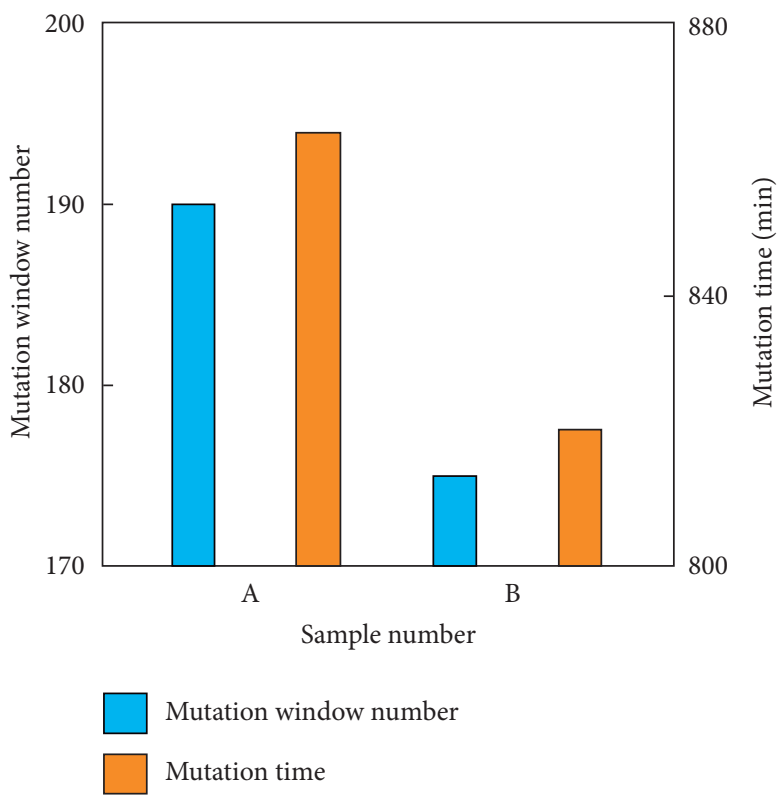

(c)

FiguRE 10: Mutation detection results of ore samples: (a) approximate entropy curves of sample A; (b) approximate entropy curves of sample B; (c) mutation results.

TABLE 2: Comparisons of the reference and measured values of self-heating initiative temperature.

\begin{tabular}{lccc}
\hline Sample number & Reference values $\left({ }^{\circ} \mathrm{C}\right)$ & Measured values $\left({ }^{\circ} \mathrm{C}\right)$ & Relative errors $(\%)$ \\
\hline $\mathrm{A}$ & 219.4 & 219.7 & 0.14 \\
$\mathrm{~B}$ & 232.3 & 239.1 & 2.84 \\
\hline
\end{tabular}

indicates that the non-linear determination method has a higher determination precision and can be used to validate the reliability of the measured results. Furthermore, so far as we know, this is a new determination method for selfheating initiative temperature of sulfide ores.

However, it should be noted that self-heating process of sulfide ores is influenced by many factors. To verify the universality of the results of this work, multi-sample experiments study under different experimental conditions will be our next tasks.

\section{Conclusions}

(1) Qualitative and quantitative identification of mutation indicate that self-heating of sulfide ores is an unsteady process. For sample A and sample B, all the Hurst exponents of different periods are more than 0.5 , which indicates that self-heating process of sulfide ores has a positive continuity. Hurst exponent of the later period is greater than that of the earlier period during the experiment. Thus, the self-heating possibility of sulfide ores can be determined according to the variation characteristics of Hurst exponent.

(2) Mutation detection results show that the mutation times of sample A and sample B are $864 \mathrm{~min}$ and $819 \mathrm{~min}$, respectively. Correspondingly, reference values of self-heating initiative temperature of the two samples are $219.4^{\circ} \mathrm{C}$ and $232.3^{\circ} \mathrm{C}$, which are in good agreement with the measured values with the average relative error of $1.49 \%$. Thus, the new determination method for self-heating initiative temperature of sulfide ores based on non-linear theory can be used to verify the reliability of the measured results.

(3) The non-linear determination method is applied to the measurement of the self-heating initiative temperature of sulfide ores, which can accurately and intuitively reflect the three stages of the self-heating of sulfide ores. It shows that the practicability and reliability of this method is of great significance to improve the theoretical and practical level of the early prevention of spontaneous combustion of sulfide ores and even to achieve the prevention and control of spontaneous combustion of sulfide ores.

\section{Data Availability}

The data used to support the findings of this study are available from the corresponding author upon request.

\section{Conflicts of Interest}

The authors declare no conflicts of interest. 


\section{Acknowledgments}

This work was supported by the National Natural Science Foundation of China (Grant nos. 51304238 and 51534008) and Key Laboratory of Ministry of Education of China for Efficient Mining and Safety of Metal Mines (Grant no. ustbmslab201805).

\section{References}

[1] S. C. Rosenblum and G. D. Spira, "Evaluation of hazard from self-heating of sulphide rock," International Journal of Rock Mechanics \& Mining Sciences \& Geomechanics Abstracts, vol. 88, no. 7, pp. 350A-351A, 1995.

[2] S. C. Bouffard and G. D. Senior, "A new method for testing the self-heating character of sulphide concentrates," Minerals Engineering, vol. 24, no. 13, pp. 1517-1519, 2011.

[3] R. A. Payant and J. A. Finch, "The effect of sulphide mixtures on self-heating," Canadian Metallurgical Quarterly, vol. 49, no. 4 , pp. $429-434,2010$.

[4] A. Iliyas, J. A. Hawboldt, and F. Khan, "Thermal stability investigation of sulfide minerals in DSC," Journal of Hazardous Materials, vol. 178, no. 1-3, pp. 814-822, 2010.

[5] B. Ngabe and J. Finch, "Self-heating: estimation of the heat release coefficient QA for $\mathrm{Ni}$ - and $\mathrm{Cu}$-concentrates and sulphide mixtures," Minerals Engineering, vol. 64, pp. 126-130, 2014.

[6] G. Sen, W. Chao, and C. Xia, "Study of risk assessment system to evaluate spontaneous combustion in mining sulfide ore," Shock and Vibration, vol. 7, no. 4, p. 409, 2005.

[7] F. Rosenblum, J. Nesset, J. E. Spira, and J. A. Finch, "Evaluation and control of self-heating in sulphide concentrates," Cim Bulletin, vol. 94, no. 1056, pp. 92-99, 2001.

[8] R. Payant, F. Rosenblum, J. E. Nesset, and J. A. Finch, "The self-heating of sulfides: galvanic effects," Minerals Engineering, vol. 26, pp. 57-63, 2012.

[9] F. Yang, C. Wu, and Y. Shi, "Factors affecting oxidation and self-heating of sulfide ores in stope," Journal of Central South University of Technology, vol. 28, no. 21, pp. 106-111, 2010.

[10] H. Liu, C. Wu, S. Ying, J. Tang, and S. Cheng, "Locating method of fire source for spontaneous combustion of sulfide ores," Journal of Central South University of Technology, vol. 18, no. 4, pp. 1034-1040, 2011.

[11] Z.-J. Pan, D.-P. Jin, Z. Liu, and X.-L. Wang, "Experimental and theoretical study on strengthening leaching of sulfide ores by surfactants," Transactions of Nonferrous Metals Society of China, vol. 137, pp. 289-299, 2020.

[12] F.-Q. Li, D. Shi, and Z.-J. Wu, "Infrared thermography for prediction of spontaneous combustion of sulfide ores," The Transactions of Nonferrous Metals Society of China, vol. 22, no. 12, pp. 3095-3102, 2012.

[13] W. Pan, H. Jin, Z. Liu, J. Tang, and S. Cheng, "Experimental and theoretical study on strengthening leaching of sulfide ores by surfactants," Process Safety and Environmental Protection, vol. 137, pp. 289-299, 2020.

[14] F. Rosenblum, J. Nesset, S. Moon, J. Finch, and K. Waters, "Reducing the self-heating of sulphides by chemical treatment with lignosulfonates," Minerals Engineering, vol. 107, pp. 78-80, 2017.

[15] P. C. Bowes, "Hazards associated with self-heating of solids," in Proceedings of Symposium on Process Industry Hazards: Accidental Release, Assessment, Contamination and Control, Institution of Chemical Engineers (Great Britain) Symposium Series, vol. 47, pp. 93-101, Glasgow, UK, 1976.
[16] M. J. Pearse, "Chemical study of oxidation of sulphide concentrates," Transactions of the Institution of Mining and Metallurgy, vol. 89, pp. C26-C36, 1980.

[17] W. F. Brinton and E. Evans, "Standardized test for evaluation of compost self-heating," Journal of Fire Sciences, vol. 36, no. 11, pp. 64-69, 1995.

[18] J. C. Jones, "Self-heating tests to establish the shipping safety of solid materials: the case for briquetted peat," Compost Science \& Utilization, vol. 14, no. 5, pp. 342-345, 1996.

[19] A. Koenig and Q. H. Bari, "Application of self-heating test for indirect estimation of respirometric activity of compost: theory and practice," Compost Science \& Utilization, vol. 8, no. 2, pp. 99-107, 2000.

[20] B. He and C. Wu, "Development of an experimental methodology for sulphide self-heating studies and the self-heating tendency of Vale's Voisey's Bay Concentrator products," Safety \& Environmental Engineering, vol. 13, no. 1, pp. 92-95, 2006.

[21] Z. Dai, "Development of an experimental methodology for sulphide self-heating studies and the self-heating tendency of Vale's Voisey's Bay Concentrator products," Minerals Engineering, vol. 92, pp. 125-133, 2016.

[22] Z. W. Xie, C. Wu, Z. J. Li, and F. Q. Yang, "Evaluation on spontaneous combustion tendency of sulfide ores based on entropy and set pair analysis theory," Journal of Central South University, vol. 43, no. 5, pp. 1858-1863, 2012.

[23] F.-Q. Yang, C. Wu, and Z. Li, "Application of uncertainty measurement model in risk evaluation on spontaneous combustion of sulfur concentrate in storehouse," Transactions of Nonferrous Metals Society of China, vol. 35, no. 2, pp. 264-268, 2010.

[24] F. Q. Yang, C. Wu, Y. Cui, and G. Lu, "Apparent activation energy for spontaneous combustion of sulfide concentrates in storage yard," Transactions of Nonferrous Metals Society of China, vol. 21, no. 2, pp. 395-401, 2011.

[25] F. Yang, C. Wu, and Z. Li, "Factors affecting oxidation and self-heating of sulfide ores in stope," Journal of Engineering Science and Technology Review, vol. 28, no. 21, pp. 106-111, 2010.

[26] H. H. Hu, Z. Liu, J. E. Li, and J. A. Cui, "Fisher discriminant analysis to the classification of spontaneous combustion tendency grade of sulphide ores," Journal of Central South University, vol. 35, no. 10, pp. 1674-1679, 2010.

[27] R. Payant, J. A. Rosenblum, J. Nesset, and J. Finch, “The selfheating of sulfides: galvanic effects," Minerals Engineering, vol. 26, pp. 57-63, 2012.

[28] F.-Q. Somot and J. Finch, "Possible role of hydrogen sulphide gas in self-heating of pyrrhotite-rich materials," Minerals Engineering, vol. 23, no. 2, pp. 104-110, 2010.

[29] F. Yang and C. Wu, "Mechanism of mechanical activation for spontaneous combustion of sulfide minerals," Transactions of Nonferrous Metals Society of China, vol. 23, no. 1, pp. 276-282, 2013.

[30] L. Li, C. Wu, and L. Wang, "Research and application of rheology-mutation theory in the prevention of sulfide ore spontaneous combustion," China Safety Science Journal, vol. 18, no. 2, pp. 81-86, 2008.

[31] W. Pan, C. Wu, Z.-J. Li, Y. Shi, and Y.-P. Yang, "Nonlinear characteristics of induced spontaneous combustion process of sulfide ores," Minerals Engineering, vol. 23, no. 12, pp. 3284-3292, 2016.

[32] F. Crundwell, "Sulfur chemistry, biofilm, and the (in) direct attack mechanism? A critical evaluation of bacterial leaching," 
Applied Microbiology and Biotechnology, vol. 9, no. 10, pp. 1081-1089, 1996.

[33] W. Sand, T. Gerke, R. Hallmann, and A. Schippers, "Sulfur chemistry, biofilm, and the (in) direct attack mechanism-a critical evaluation of bacterial leaching," Applied Microbiology and Biotechnology, vol. 43, no. 6, pp. 961-966, 1995.

[34] L. Li, B. S. Wu, F. Yang, and G. Ge, "Study on Relevant Factors during Measuring Sulfide Ore Pile Temperature in the State of Self-Heating by Infrared Spectroscopy," Fire Safety Science, vol. 17, no. 1, (in Chinese), 2008.

[35] S. Biswas and B. S. Górski, "Time-dependent vibrational spectral analysis of first principles trajectory of methylamine with wavelet transform," Mechanical Systems and Signal Processing, vol. 19, no. 15, pp. 9912-9922, 2017.

[36] G. Litak, G. Gorski, R. Mosdorf, and A. Rysak, "Study of dynamics of two-phase flow through a minichannel by means of recurrences," Mechanical Systems \& Signal Processing, vol. 89, pp. 48-57, 2017.

[37] R. D. Baker, "A methodology for sensitivity analysis of models fitted to data using statistical methods," IMA Journal of Management Mathematics, vol. 12, no. 1, pp. 23-39, 2001.

[38] D. L. Sampaio, R. Nicoletti, F. Liu, and Z. Xue, "Detection of cracks in shafts with the Approximated Entropy algorithm," Insight-Non-destructive Testing and Condition Monitoring, vol. 72-73, pp. 286-302, 2016.

[39] W. Li, X. Di, S. Bai, F. Liu, and Z. Xue, "Feature analysis of metal magnetic memory signals for weld cracking - based on wavelet energy spectrum," Insight-Non-Destructive Testing and Condition Monitoring, vol. 48, no. 7, pp. 426-429, 2006.

[40] F. Takens, "Determing strange attractors in turbulence," Lecture Notes in Math, vol. 898, pp. 361-381, 1981.

[41] J. Lin and Y. Wang, "Selection of time delay in speech signal phase space reconstruction complex autocorrelation method," Signal Processing, vol. 15, no. 3, pp. 220-225, 1999.

[42] H. Wang and S. Lu, Nonlinear Time Series Analysis and its Application, Science Press, Beijing, China, (in Chinese), 2006.

[43] D. Yu, D. Zhao, and Y. Wang, "Chaotic dynamics of propeller singing," Acta Acustica, vol. 35, no. 5, pp. 530-538, 2010.

[44] H. Chen, C. Hong, and Y. Li, "Self-similar traffic: Hurst parameter estimation based on multiresolution sampling and wavelet analysis," Acta Electronica Sinica, vol. 26, no. 7, pp. 88-93, 1998. 